\title{
Estrogen Restores Multisynaptic Boutons in the Dorsolateral Prefrontal Cortex while Promoting Working Memory in Aged Rhesus Monkeys
}

\author{
(D)Yuko Hara, ${ }^{1,2}$ Frank Yuk, ${ }^{1,2}$ Rishi Puri, ${ }^{1,2}$ William G. M. Janssen, ${ }^{1,2}$ Peter R. Rapp, ${ }^{5}$ and John H. Morrison ${ }^{1,2,3,4,6,7}$ \\ ${ }^{1}$ Fishberg Department of Neuroscience and Kastor Neurobiology of Aging Laboratories, ${ }^{2}$ Friedman Brain Institute, ${ }^{3}$ Department of Geriatrics and Palliative \\ Medicine, and ${ }^{4}$ Graduate School of Biomedical Sciences, Icahn School of Medicine at Mount Sinai, New York, New York 10029, ${ }^{5}$ National Institute on Aging, \\ Laboratory of Behavioral Neuroscience, Baltimore, Maryland 21224, ${ }^{6}$ California National Primate Research Center, Davis, California 95616, and \\ ${ }^{7}$ Department of Neurology, School of Medicine, University of California, Davis, California 95616
}

Humans and nonhuman primates are vulnerable to age- and menopause- related decline in working memory, a cognitive function reliant on area 46 of the dorsolateral prefrontal cortex (dlPFC). We showed previously that presynaptic mitochondrial number and morphology in monkey dlPFC neurons correlate with working memory performance. The current study tested the hypothesis that the types of synaptic connections these boutons form are altered with aging and menopause in rhesus monkeys and that these metrics may be coupled with mitochondrial measures and working memory. Using serial section electron microscopy, we examined the frequencies and characteristics of nonsynaptic, single-synaptic, and multisynaptic boutons (MSBs) in the dIPFC. In contrast to our previous observations in the monkey hippocampal dentate gyrus, where MSBs comprised $\sim 40 \%$ of boutons, the vast majority of dlPFC boutons were single-synaptic, whereas MSBs constituted a mere $10 \%$. The frequency of MSBs was not altered by normal aging, but decreased by over $50 \%$ with surgical menopause induced by ovariectomy in aged monkeys. Cyclic estradiol treatment in aged ovariectomized animals restored MSB frequencies to levels comparable to young and aged premenopausal monkeys. Notably, the frequency of MSBs positively correlated with working memory scores, as measured by the average accuracy on the delayed response (DR) test. Furthermore, MSB incidence positively correlated with the number of healthy straight mitochondria in dIPFC boutons and inversely correlated with the number of pathological donut-shaped mitochondria. Together, our data suggest that MSBs are coupled to cognitive function and mitochondrial health and are sensitive to estrogen.

Key words: area 46; delayed response; estradiol; menopause; mitochondria; MSB

Significance Statement

Many aged menopausal individuals experience deficits in working memory, an executive function reliant on recurrent firing of prefrontal cortex (PFC) neurons. However, little is known about the organization of presynaptic inputs to these neurons and how they may be altered with aging and menopause. Multisynaptic boutons (MSBs) were of particular interest, because they form multiple synapses and can enhance coupling between presynaptic and postsynaptic neurons. We found that higher MSB frequency correlated with better working memory performance in rhesus monkeys. Additionally, aged surgically menopausal monkeys experienced a 50\% loss of MSBs that was restored with cyclic estradiol treatment. Together, our findings suggest that hormone replacement therapy benefits cognitive aging, in part by retaining complex synaptic organizations in the PFC.

\section{Introduction}

Working memory is an online, short-term, and flexible information processing capacity that enables adaptive decision making and goal-directed behavior (Goldman-Rakic, 1995; Arnsten et al., 2012). This executive function is highly vulnerable to the effects of aging and menopause in humans and nonhuman pri- 
mates and can be assessed in rhesus monkeys using the wellcharacterized delayed response (DR) test of visuospatial working memory (Bartus et al., 1978; Rapp and Amaral, 1989; Bachevalier et al., 1991; Oscar-Berman et al., 1992; Roberts et al., 1997; Voytko and Tinkler, 2004). In rhesus monkeys, DR performance is reliant on the integrity of the dorsolateral prefrontal cortex (dlPFC) area 46 (Funahashi et al., 1989; Goldman-Rakic, 1995), a brain region that shares striking anatomical and functional homology to the human area 46 (McCarthy et al., 1994; Petrides and Pandya, 1999). Rhesus monkeys are valuable models of human aging and menopause, because their neuronal gene expression, reproductive physiology, and patterns of endocrine senescence closely resemble those of humans (Matt et al., 1998; Gill et al., 2002; Woller et al., 2002; Nichols et al., 2005; Loerch et al., 2008; Walker and Herndon, 2008; Hara et al., 2012a).

Area 46 neurons exhibit persistent firing while spatial information is held in working memory (Funahashi et al., 1989), and this process is highly energy demanding. We reported previously that mitochondrial count and morphology in presynaptic inputs to dlPFC neurons are altered with aging and menopause and correlate with working memory in rhesus monkeys (Hara et al., 2014). Specifically, higher working memory scores were associated with greater numbers of healthy straight mitochondria in dlPFC boutons and a lower incidence of boutons harboring malformed donut-shaped mitochondria, which exhibited abnormally small synaptic contacts.

Presynaptic boutons are plastic structures and undergo morphological and synaptic remodeling, which affect neuronal circuitry and function (Gogolla et al., 2007; Soghomonian et al., 2010). In contrast to a dendritic spine, which typically receives synaptic contact from a single bouton, a bouton can form a single synapse, multiple synapses, or no synapse at all. Furthermore, the specific type (e.g., axodendritic vs axospinous) and number of contacts a bouton forms provides important information about the complexity and strength of synaptic connections. In particular, multisynaptic boutons (MSBs) may act as a morphological substrate to enhance coupling between presynaptic and postsynaptic neurons (Harris, 1995; Toni et al., 1999; Geinisman et al., 2001; Yankova et al., 2001). In rats and monkeys, hippocampal MSBs are associated with memory functions and are responsive to hormonal status (Geinisman et al., 2001; Hara et al., 2011). However, little is known about the roles of MSBs in the PFC, particularly in primates.

The current study tested the hypothesis that the types of synaptic connections these dlPFC boutons form are altered with aging or menopause in rhesus monkeys and that these metrics are coupled with mitochondrial measures and working memory. Our results reveal that the frequency of MSBs is correlated with working memory, associated with mitochondrial morphology, and responsive to estrogen.

\section{Materials and Methods}

Monkeys. Rhesus monkeys (Macaca mulatta; total, $n=29$ ) were housed in colonies of $\sim 40$ individuals at the California National Primate Research Center at University of California, Davis. Experimental treatments, behavioral data, perfusion protocol, and tissue preparation for monkeys included in this study have been described in detail previously (Rapp et al., 2003; Hao et al., 2007; Dumitriu et al., 2010; Hara et al.,

The authors declare no competing financial interests.

Correspondence should be addressed to Dr. John H. Morrison, California National Primate Research Center, University of California at Davis, 1 Shields Avenue, Davis, CA 95616. E-mail: jhmorrison@ucdavis.edu.

DOI:10.1523/JNEUROSCI.3480-13.2016

Copyright $\odot 2016$ the authors $\quad 0270-6474 / 16 / 360902-10 \$ 15.00 / 0$
2014). All experiments were conducted in compliance with the NIH Guide for the Care and Use of Laboratory Animals and were approved by the Institutional Care and Use Committee at the University of California, Davis.

Surgically intact monkeys. The surgically intact cohort comprised 7 young adult $(10.4 \pm 0.5$ years $)$ and 14 aged $(29.0 \pm 1.1$ years $)$ female rhesus monkeys. None of these monkeys received prior invasive or pharmacological manipulations expected to influence the cognitive or neurobiological measures examined here. Menses status in aged subjects was determined by daily inspection for vaginal bleeding for 2 years before perfusion. The average life span of captive rhesus monkeys is under 25 years, and human age equivalence is estimated at a 1:3 ratio (Tigges et al., 1988). Menopause in rhesus monkeys typically occurs by age 27 years, later in life relative to humans (Gilardi et al., 1997; Walker and Herndon, 2008). A liberal cycle length (interval between the first days of menses) of $24-45 \mathrm{~d}$ was used to classify monkeys as premenopausal, as described previously (Hara et al., 2012b). Because the transition between perimenopausal and postmenopausal statuses is difficult to determine with precision, we adopted the convention of an earlier study (Gilardi et al., 1997) and classified monkeys in which the cycle length exceeded $45 \mathrm{~d}$ as perimenopausal/postmenopausal. Ovarian status was confirmed by urinalysis of hormonal data for 16 out of 21 monkeys (Hara et al., 2012c).

Ovariectomized monkeys. Eight aged female monkeys ( $22 \pm 0.6$ years) classified as premenopausal and perimenopausal underwent bilateral ovariectomy (OVX) and were randomly assigned to age-matched vehicle $(\mathrm{OVX}+\mathrm{V}, n=4)$ and estradiol (OVX $+\mathrm{E}, n=4)$ groups, as described in detail previously (Rapp et al., 2003). Briefly, subjects were injected with ketamine $(10 \mathrm{mg} / \mathrm{kg}$, i.m. $)$ and atropine $(0.04 \mathrm{mg} / \mathrm{kg}$, s.c. $)$, intubated, and placed on isoflurane anesthesia. A ventral midline incision was made and the ovarian vessels and fallopian tubes were isolated, ligated, and severed. After the ovaries were removed, the abdominal wall was closed. Monkeys were observed until responsive, and oxymorphone was administered for postoperative pain $(1.5 \mathrm{mg} / \mathrm{kg}$, i.m., three times per day for $2 \mathrm{~d})$. After a post-OVX interval of $30 \pm 1.7$ weeks, $100 \mu \mathrm{g}$ of estradiol cypionate (Pharmacia) in $1 \mathrm{ml}$ of peanut oil $(\mathrm{OVX}+\mathrm{E})$ or $1 \mathrm{ml}$ of peanut oil alone $(\mathrm{OVX}+\mathrm{V})$ was injected intramuscularly every 3 weeks throughout $2-3$ years of behavioral testing. Injections were administered by experimenters blind to the group designation of the monkeys.

Behavioral testing. The DR test of visuospatial working memory was conducted as described in detail previously (Rapp et al., 2003). Monkeys watched from behind a transparent screen while the left or right well of the test tray was baited with a food reward. Then, both wells were covered and the retention interval was imposed by lowering an opaque screen. When the opaque screen was lifted at the end of the delay interval, the subject retrieved the reward if the correct location was chosen. During the acquisition phase, the screen was raised immediately $(0 \mathrm{~s})$, and the monkey continued on this phase until a criterion of over $90 \%$ accuracy was achieved. Next, a retention interval of $1 \mathrm{~s}$ was used and when the monkey reached $90 \%$ correct or better, the acquisition phase ended. To test working memory performance, successively longer retention intervals of $5,10,15,30$, and $60 \mathrm{~s}$ were imposed ( 90 trials total at each delay, 30 trials/d). The intertrial interval was set at $20 \mathrm{~s}$, and a white-noise generator masked extraneous sounds during testing.

In addition to DR, tasks reliant on medial temporal lobe structures such as the delayed nonmatching-to-sample test of recognition memory and the two-choice object discrimination test were also conducted as described previously in detail (Rapp et al., 2003).

In cases where perfusion could not be scheduled soon after completion of behavioral testing, monkeys continued mock training until the day before perfusion to avoid testing-induced variability in morphological measures. ANOVA showed that there were no significant differences across groups in the amount of time lapsed between the end of behavioral testing and the perfusion date $(p>0.05)$. Furthermore, no correlations were found between the amount of time lapsed and any of the behavioral measures obtained $(p>0.05)$.

Perfusion and tissue processing. Monkeys were perfused as described previously (Hara et al., 2014). Briefly, subjects were deeply anesthetized with ketamine hydrochloride $(25 \mathrm{mg} / \mathrm{kg})$ and pentobarbital $(20-35 \mathrm{mg} / \mathrm{kg}$, i.v.), intubated, and mechanically ventilated. After the 
left ventricle of the heart was injected with $1.5 \mathrm{ml}$ of $0.5 \%$ sodium nitrate, the descending aorta was clamped. Surgically naive monkeys were perfused transcardially with ice cold $1 \%$ paraformaldehyde in $0.1 \mathrm{~m}$ phosphate buffer (PB), $\mathrm{pH} 7.2$, for $2 \mathrm{~min}$, followed by $4 \%$ paraformaldehyde in $0.1 \mathrm{M} \mathrm{PB}$ at $250 \mathrm{ml} / \mathrm{min}$ for $10 \mathrm{~min}$, after which the flow rate was decreased to $100 \mathrm{ml} / \mathrm{min}$ for $50 \mathrm{~min}$. Aged ovariectomized monkeys (OVX $+\mathrm{V}$, OVX $+\mathrm{E})$ were perfused with cold $1 \%$ paraformaldehyde/PB for $1 \mathrm{~min}$ at $180 \mathrm{ml} / \mathrm{min}$, followed by $4 \%$ paraformaldehyde/PB for $12 \mathrm{~min}$ at $200 \mathrm{ml} / \mathrm{min}$. We were unable to add glutaraldehyde in our perfusate, because the monkey brains were also used in multidisciplinary collaborative studies where the protocols were incompatible with glutaraldehyde fixation. Instead, we used glutaraldehyde during postfixation before embedding, which allowed sufficient preservation of ultrastructural morphology that was consistent across monkeys. For postfixation, the PFC subregion including the principal sulcus (area 46) was immersed for $6 \mathrm{~h}$ in $4 \%$ paraformaldehyde/PB with $0.125 \%$ glutaraldehyde. The subregion was rinsed in $\mathrm{PB}$, and sectioned at 50 - and $400-\mu \mathrm{m}$-thick sections on a vibratome (Leica) for confocal and electron microscopy, respectively.

Confocal microscopic analysis of lipofuscin. General oxidative damage in area 46 layer III was assessed using lipofuscin fluorescence intensity as described previously (Hara et al., 2014). Because the $50 \mu \mathrm{m}$ slices were heavily used and depleted in previous stereological and immunocytochemical studies for many of the subjects, lipofuscin analysis could only be performed in 8 of the 21 surgically intact monkeys. The $50 \mu \mathrm{m}$ sections containing area 46 were mounted on glass slides and coverslipped with Vectashield mounting medium (Vector Laboratories). Images of autofluorescent lipofuscin in layer III of area 46 were obtained using a Zeiss LSM 780 inverted microscope with an Argon 488 -nm wavelength set at $2 \%$ laser output, a $40 \times / 1.46$ numerical aperture objective set to a digital zoom of $0.6 \times$, and pixel resolution set to $1024 \times 1024$ (12 bit), and line-averaged four times at a pixel dwell of $3.15 \mu \mathrm{s}$. All images were taken at a depth of $10 \mu \mathrm{m}$ from the tissue surface and were adjusted for similar gain and offset values. Three images from area 46 layer III (total area, $0.378 \mathrm{~mm}^{2}$ ) were taken for each of the eight animals. Images were imported into Adobe Photoshop version CS5 (Adobe Systems), and mean lipofuscin intensity per pixel was calculated as the average of the three raw images, after subtracting background intensity (mean intensity of nine blood vessels or nuclei lacking lipofuscin).

Embedding for electron microscopy. Freeze substitution and lowtemperature embedding of sections from all 29 monkeys were performed as described previously in detail (Hara et al., 2012b). Briefly, $400-\mu \mathrm{m}$-thick sections were immersed in increasing concentrations of cryoprotectant $(10,20$, and $30 \%$ glycerol in PB) and then plunged in liquid propane cooled by liquid nitrogen $\left(-190^{\circ} \mathrm{C}\right)$ in a Universal Cryofixation System KF80 (Reichert-Jung). Sections were immersed in $1.5 \%$ uranyl acetate in anhydrous methanol $\left(-90^{\circ} \mathrm{C}\right)$ for $24 \mathrm{~h}$ in a cryosubstitution Automatic Freeze-Substitution System unit (Leica). The temperature was raised $4^{\circ} \mathrm{C} / \mathrm{h}$ from -90 to $-45^{\circ} \mathrm{C}$. At $-45^{\circ} \mathrm{C}$, the sections were washed with anhydrous methanol and infiltrated with Lowicryl HM20 resin (Electron Microscopy Sciences), with a progressive increase in the resin to methanol ratio for $1 \mathrm{~h}$ each, followed by pure Lowicryl overnight. Resin polymerization was achieved with UV light $(360 \mathrm{~nm})$ at $-45^{\circ} \mathrm{C}$ for $48 \mathrm{~h}$, followed by $24 \mathrm{~h}$ at room temperature. Fifteen or more consecutive ultrathin sections were cut into 80 -nm-thick sections using a Diatome diamond knife and mounted on each formvar/carbon-coated slot grid (Electron Microscopy Sciences).

Electron microscopic analysis of axonal boutons and residing mitochondria. All electron microscopic imaging and axonal/mitochondrial analyses were performed by the same examiner, blind to the group designation and behavioral performance. For each of the 29 monkeys, five series of 15 consecutive ultrathin sections were imaged at $2500 \times$ on a Hitachi H-7700 electron microscope (Hitachi High Technologies America) with an AMT Advantage CCD camera (Advanced Microscopy Techniques), using a systematic-random approach. To ensure that images were taken from layer III of area 46, images were captured only between 250 and $350 \mu \mathrm{m}$ from the layer I (acellular) and II (cell-rich) intersection, and areas that contained cell bodies and blood vessels were excluded. The electron micrographs were adjusted for brightness and sharpness with Adobe Photoshop (version CS5). Three-dimensional reconstructions of a subset of axonal boutons, presynaptic mitochondria, and associated postsynaptic partners were performed using Reconstruct, an open source software developed by Dr. Kristen Harris (University of Texas at Austin, Austin, TX) and her laboratory (version 1.1.0.0; http://synapses.clm.utexas.edu).

First, an unbiased stereological approach was used to measure area 46 bouton density. For each series, a square grid of $2.29 \times 2.29 \mu \mathrm{m}$ was drawn using the Photoshop ruler guide tool (Adobe Systems), which applied to all 15 sections. Boutons were identified across the 15 serial sections and counted if (1) they were fully contained within the volume examined or (2) if they were transected by section 1 and/or the left grid and/or the bottom grid, while not coming in contact with any of the following: section 15, the right grid, or the top grid. The total volume examined per monkey for this volumetric analysis was $31.46 \mu \mathrm{m}^{3}$, and the total number of boutons included in this analysis for each experimental group was 130 for the young premenopausal group, 81 for the aged premenopausal group, 159 for the aged perimenopausal/postmenopausal group, 77 for the aged OVX $+\mathrm{V}$ group, and 69 for the aged $\mathrm{OVX}+\mathrm{E}$ group.

Next, analysis of presynaptic connectivity was performed as described in our prior work (Hara et al., 2011) and was consistent with methods used by other groups (Woolley et al., 1996). Unlike dendritic spines with electron-dense postsynaptic densities, axon terminals do not contain a visually distinct counting unit that can be used in a traditional unbiased disector method. Therefore, we used the middle eighth section in each series as a reference section (Fig. 1) and all presynaptic boutons containing at least three synaptic round vesicles that were transected in this middle section were identified and analyzed. This method allowed us to follow each bouton to its fullest extent across 15 serial sections, where every single synaptic contact and mitochondria could be accurately identified in the vast majority of cases. While this method is subject to bias, the density of boutons captured in the eighth section strongly correlated with the volumetric bouton density obtained stereologically (Pearson $r=$ $0.545 ; p=0.011$ ). Thus, the bias was expected to be largely consistent across subjects. On average, 150 boutons were identified and analyzed per monkey using this approach. Each bouton was followed throughout the 15 serial sections and the following morphological characteristics were noted: (1) the number of synaptic contacts it formed [0, nonsynaptic boutons (NSBs); 1, single-synaptic boutons (SSBs); 2+, MSBs]; (2) the type of synaptic contact it formed (axospinous vs axodendritic); and (3) the number and morphology of mitochondria it contained. Synaptic contacts were not classified further into asymmetric (excitatory) or symmetric (inhibitory) configurations; however, it is well established that the great majority $(\sim 87 \%)$ of boutons forming synapses in this brain region are asymmetric (Peters et al., 2008). Presynaptic mitochondria were classified into three basic shapes (straight, curved, and donut), as described previously (Hara et al., 2014). Curved mitochondria were bent $>90$ degrees, while donut (toroidal) mitochondria were ring shaped. All other mitochondria were classified as straight. Volumes of 50 randomly chosen boutons per monkey were estimated by determining the cross-sectional areas of the boutons using the Adobe Photoshop lasso tool (Adobe Systems) in each section and multiplying the total area by the section thickness. In all monkeys examined, regardless of age, menses status, or treatment, only $6-14 \%$ of area 46 boutons extended beyond the 15 serial sections used in the analyses. Because the proportions of boutons extending beyond the 15 serial sections were similar across groups (ANOVA, $p>0.05$ ), all boutons were included in the analyses so as not to preferentially exclude large boutons. The total volume of area 46 layer III examined per monkey for these synaptic and mitochondrial analyses was $517.84 \mu \mathrm{m}^{3}$.

Statistical analyses. SPSS 11.0 was used for all statistical analyses. The behavioral and morphological data were not significantly different from a normal distribution ( $p>0.05$, one-sample KolmogorovSmirnov test), and, accordingly, parametric statistics were used to test for potential differences across groups. One-way and repeated measures ANOVA were used to determine whether cognitive task acquisition and accuracy differed across groups according to age, menses 

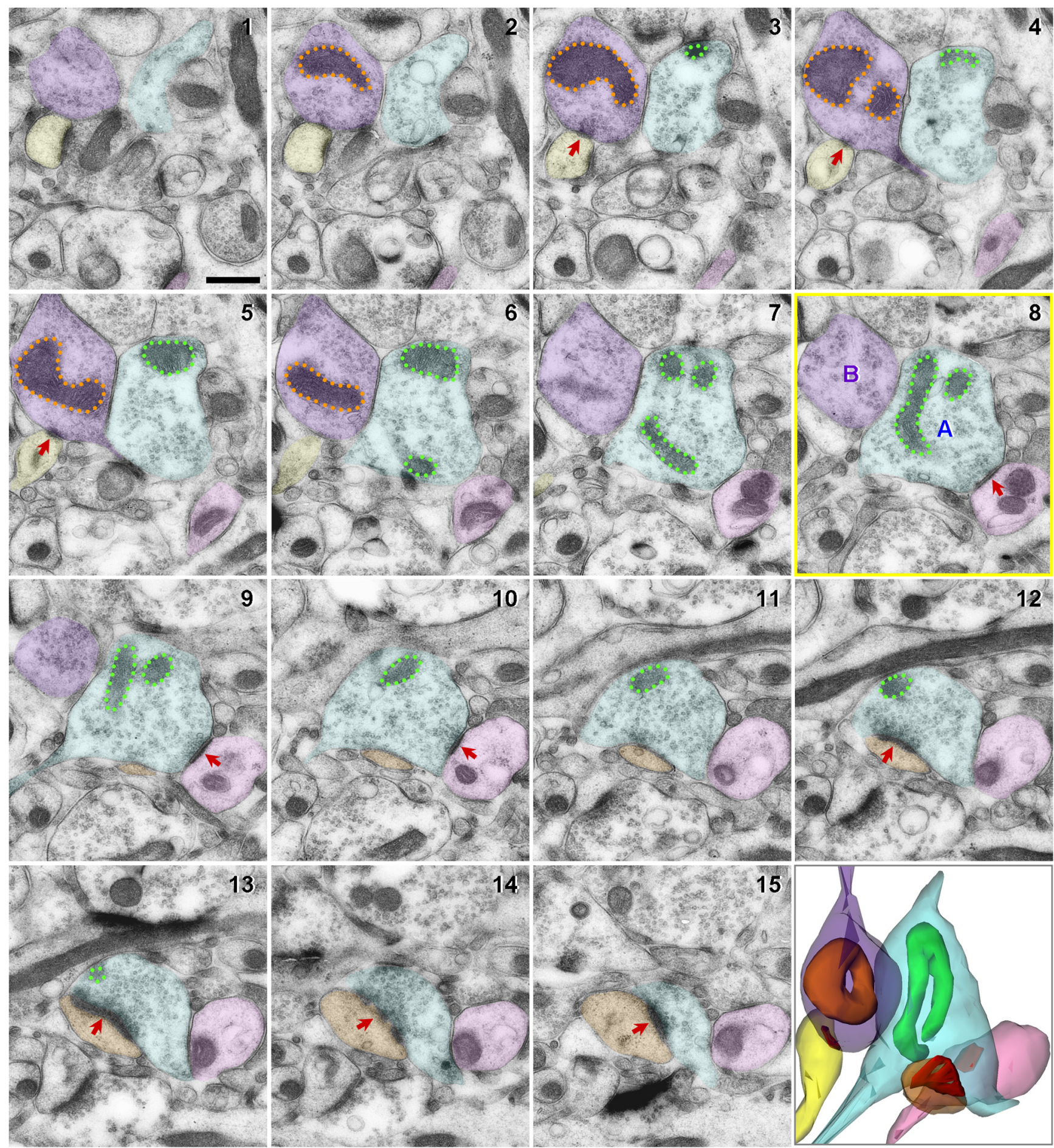

Figure 1. Characterization of area 46 axonal boutons and residing mitochondria using 15 serial section electron micrographs and 3D reconstruction. The middle section (8) outlined in yellow was used as a reference section. Presynaptic boutons transected in this middle section were identified and followed throughout the series. All excitatory asymmetric synaptic contacts consisting of a thick postsynaptic density (PSD; red arrows) were marked. Bouton A (blue) contains a curved mitochondrion (green) and forms multisynaptic contacts with a dendritic shaft (pink, sections 1-15) and a dendritic spine with a perforated PSD (orange, sections 9-15). Bouton B (purple) contains a donut-shaped mitochondrion (orange) and forms a single-synaptic contact with a dendritic spine (yellow, sections 1-6). Scale bar, $500 \mathrm{~nm}$.

status, or treatment. Multivariate ANOVA (MANOVA) followed by Tukey's post hoc test probed potential effects of group morphological measures while controlling for multiple comparisons. The surgically intact cohort and the ovariectomized cohort were analyzed separately for group comparisons to examine each variable (age, menses status, treatment) separately without overcorrecting for multiple comparisons. Two-way MANOVA, followed by Tukey's post hoc test was used to determine whether morphological measures differed between groups or by types of synaptic contact. Pearson correlations were used to evaluate potential relationships between morphological indices and behavioral measures. Correlations that were significant across all subjects were further assessed within young and aged groups separately to test whether correlations persisted independent of age. The $\alpha$ level was set at 0.05 for statistical significance. Observed power in 

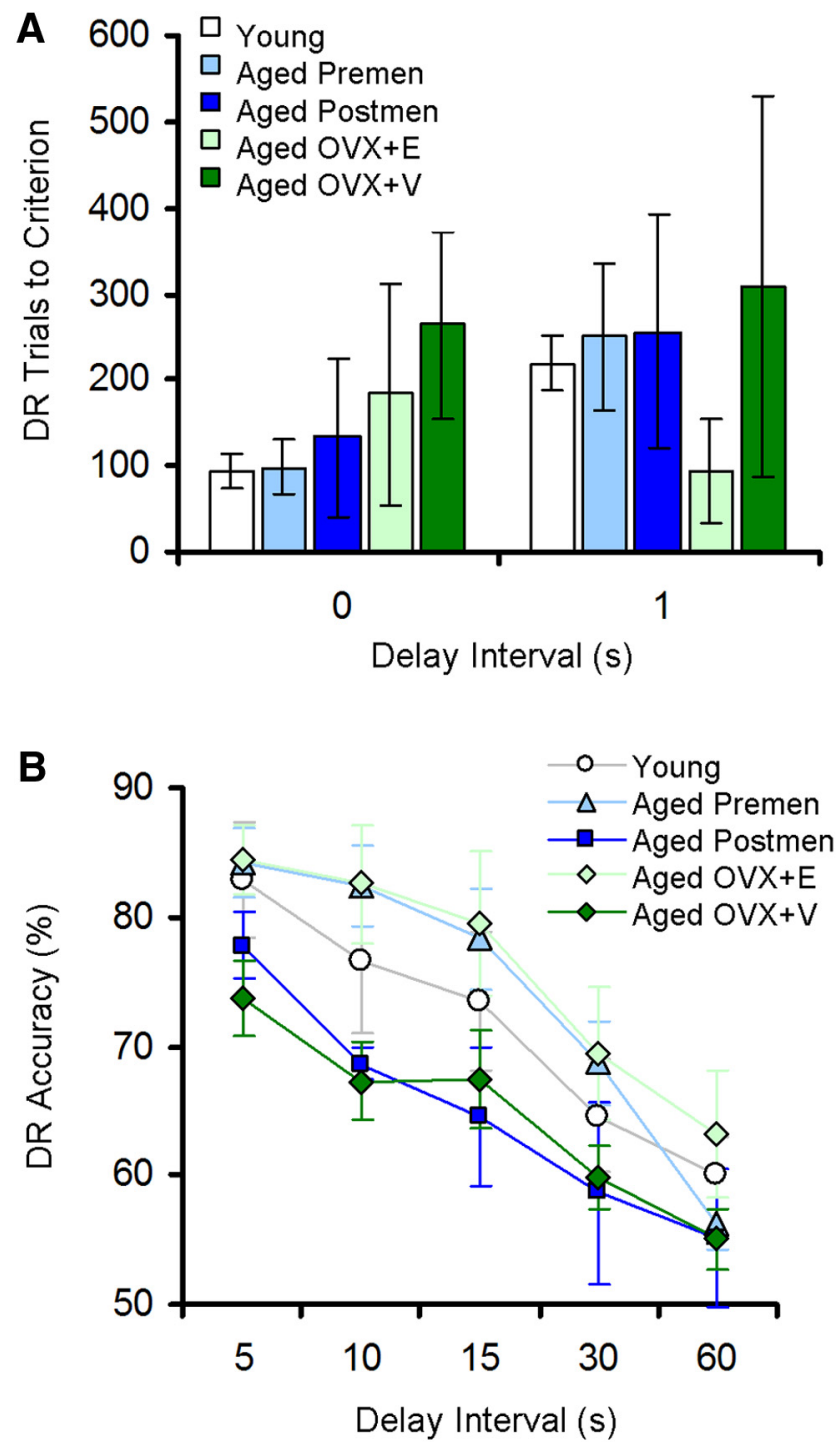

Figure 2. Behavioral measures across groups designated by age, menses status, and treatment. $\boldsymbol{A}$, Number of trials to reach the DR acquisition criterion at 0 and $1 \mathrm{~s}$ delay intervals. $\boldsymbol{B}$, Average DR accuracy across delay intervals of $5-60$ s. Young, $n=7$; aged premenopausal, $n=$ 5; aged postmenopausal, $n=9$; aged $0 \mathrm{VX}+\mathrm{E}, n=7$; aged $0 \mathrm{VX}+\mathrm{V}, n=7$. Data are expressed as mean \pm SEM.

ANOVA were determined to confirm that the sample size was sufficient to support the data.

\section{Results}

\section{Behavioral characterization}

DR data for all of the monkeys included in the present study have been presented in detail previously (Rapp et al., 2003; Dumitriu et al., 2010; Hara et al., 2014). No significant effects of age, menses status, or treatment were observed with the number of trials to reach task acquisition criterion of $90 \%$ or above accuracy $(p>$ 0.05 ; Fig. $2 A$ ), consistent with the literature reporting that aged and menopausal monkeys are able to acquire the task and are minimally impaired at very short delays of 0 and $1 \mathrm{~s}$ (Rapp and Amaral, 1989; Rapp et al., 2003). When working memory was subsequently challenged with successively longer delays (5-60 s), repeated measures ANOVA showed a significant main effect of delay $\left(F_{(4,96)}=53.109, p=2.34 \times 10^{-25}\right.$, observed power $=$ $1.000)$ and a trend for a main group effect $\left(F_{(4,96)}=2.322, p=\right.$ $0.08)$ in the absence of a group by delay interaction ( $p>0.05$; Fig.
$2 B)$. The group effect and post hoc tests did not reach statistical significance due to a large variability within groups and an overall poorer than expected performance of young subjects compared to what has been reported previously (Bachevalier et al., 1991; Rapp et al., 2003). However, naturally and surgically menopausal $($ aged OVX $+\mathrm{V})$ monkeys performed numerically worse than premenopausal and estradiol-treated (Aged OVX+E) monkeys (Fig. 2B), consistent with previous findings (Roberts et al., 1997).

\section{Menopause-related differences in synaptic characteristics of area 46 axonal boutons}

As recurrent firing of dlPFC neurons is critical for working memory (Funahashi et al., 1989; Goldman-Rakic, 1995), we examined whether characteristics of presynaptic inputs to these neurons are altered with aging, menopause, or estrogen treatment. First, an unbiased stereological approach was used to determine bouton density per volume for each subject. One-way ANOVA showed a lack of age, menses status, or treatment effect on the total number of boutons observed per unit volume (ANOVA, $p>0.05$ for each condition; Fig. $3 A$ ).

We next examined whether types of axonal contacts in area 46 differed by age, menses status, or treatment. In all groups, the vast majority of boutons made single-synaptic contacts (SSBs; $>80 \%$ ), whereas NSBs and MSBs were less frequently observed. In the ovary-intact cohort, multivariate ANOVA revealed a significant menopause effect in the percentage of NSBs such that aged perimenopausal/postmenopausal monkeys had a significantly higher percentage compared to aged premenopausal monkeys $\left(F_{(2,36)}=3.934 ; p=0.038\right.$; observed power, 0.631 ; Tukey's post hoc test, $p=0.030$; Fig. $3 A)$. No group effects were observed in the frequency of SSBs $(p>0.05)$. In the aged ovariectomized cohort, a treatment effect was observed in the percentage of MSBs such that vehicle-treated monkeys had approximately half the proportion of MSBs compared to estradiol-treated monkeys $\left(F_{(1,6)}=13.825 ; p=0.01\right.$; observed power, 0.87 ; Fig. $\left.3 B\right)$.

We showed previously that normal aging and surgical menopause result in significant reductions in dendritic spines on layer III area 46 neurons (Hao et al., 2007; Dumitriu et al., 2010). Therefore, we classified MSBs based on whether they formed axospinous or axodendritic contacts to examine whether one type was more vulnerable to the effects of aging or menopause than the other. Although multivariate ANOVA failed to show age or natural menopause effects $(p>0.05)$, a significant treatment effect was observed in the density of MSBs forming one or more axospinous contacts such that vehicle-treated monkeys had only half of the density of axospinous MSBs compared to estradioltreated monkeys $\left(F_{(1,6)}=17.643 ; p=0.006\right.$; observed power, 0.935; Fig. 3C). The densities of MSBs forming one or more axodendritic contacts were not significantly different across age, menses status, or treatment groups $(p>0.05)$.

We then examined whether morphological characteristics of NSBs, SSBs, and MSBs are different from one another. A two-way MANOVA showed a significant main effect of synaptic contact type (NSB, SSB, and MSB) on axonal bouton volume $\left(F_{(8,168)}=\right.$ $19.791 ; p=5.39 \times 10^{-7}$; observed power, 1.000) and average mitochondrial count $\left(F_{(8,168)}=41.21 ; p=1.32 \times 10^{-12}\right.$; observed power, 1.000; Fig. $3 D$ ) in the absence of a group effect or a group by synaptic type interaction $(p>0.05)$. Tukey's post hoc tests revealed that MSBs were significantly larger and contained more mitochondria than SSBs (volume, $p=0.006$; mitochondria, $p=3.94 \times 10^{-7}$ ) or NSBs (volume, $p=1.29 \times 10^{-8}$; mitochondria, $p=5.10 \times 10^{-9}$ ), and SSBs were larger and contained more mitochondria than NSBs (volume, $p=0.0002$; mi- 


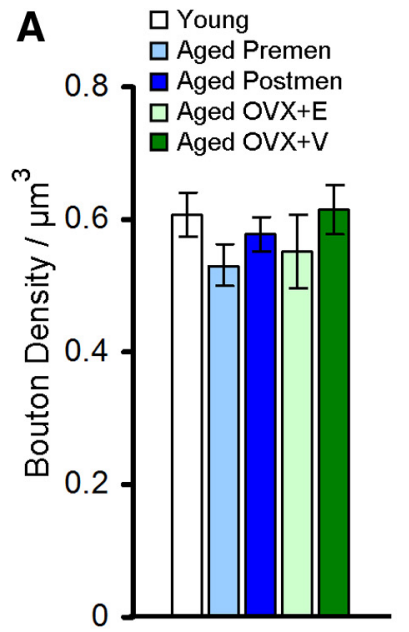

B

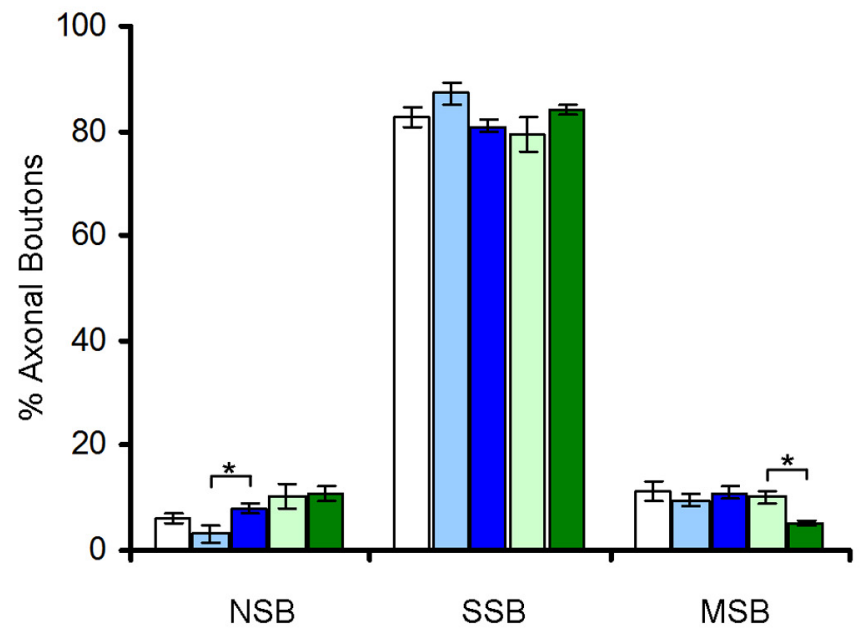

E
C

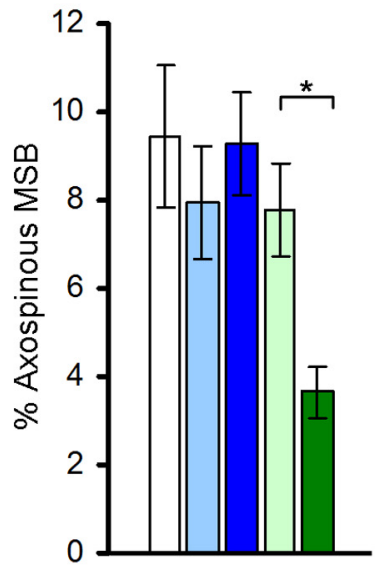

D

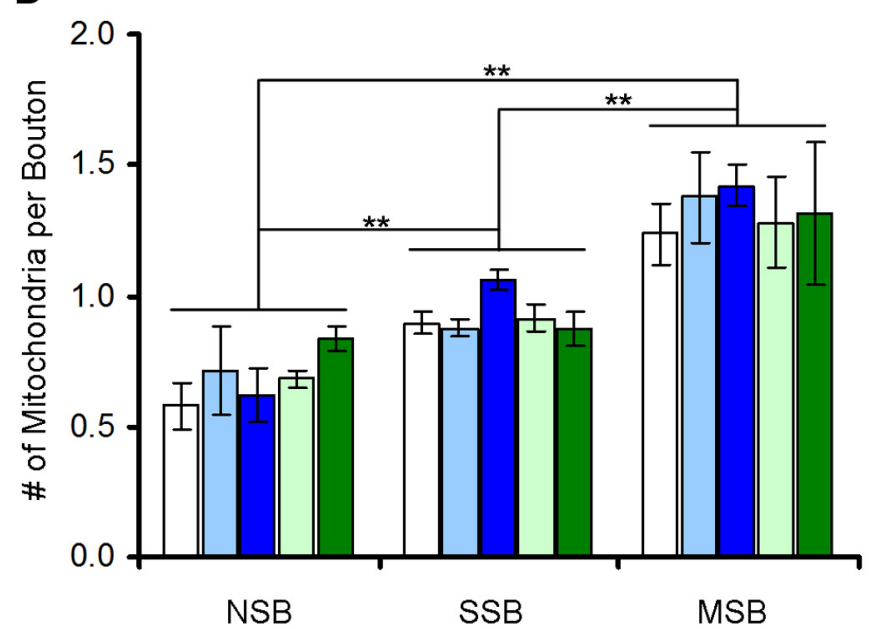

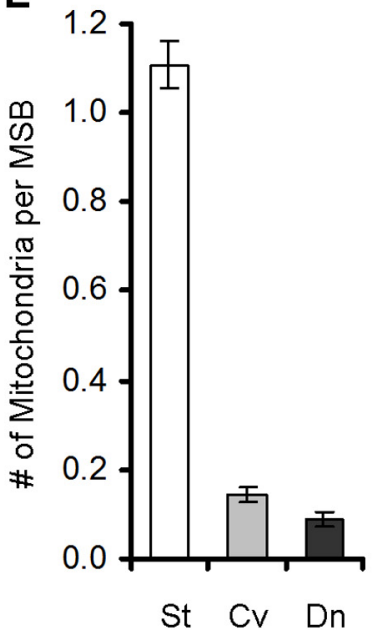

Figure 3. Synaptic characteristics of monkey area 46 axonal boutons by age, menses status, and treatment. $A$, Area 46 bouton density per cubic micrometer. $B$, Proportions of NSBs, SSBs, and MSBs. C, The areal density of MSBs contacting one or more dendritic spines. D, The average number of mitochondria in NSBs, SSBs, and MSBs. $\boldsymbol{E}$, The average number of straight (St), curved (Cv), and donut-shaped (Dn) mitochondria in MSBs. Data are expressed as group means \pm SEM for $\boldsymbol{A}-\boldsymbol{D}$, and means \pm SEM collapsed across groups for $\boldsymbol{E}$. Young, $n=7$; aged premenopausal, $n=5$; aged postmenopausal, $n=9$; aged $0 \mathrm{VX}+\mathrm{E}, n=4$; aged $\mathrm{OVX}+\mathrm{V}, n=4 .{ }^{*} p<0.05 ;{ }^{* *} p<0.01$.

tochondria, $p=0.0003)$. Regardless of age, menses status, or treatment (group effect, $p>0.05$ ), MSBs contained predominantly straight mitochondria $(82.8 \%)$, whereas curved $(10.6 \%)$ and donut-shaped mitochondria $(6.6 \%)$ were infrequently observed (Fig. 3E).

The average number of synaptic contacts per MSB was not significantly different across age, menses status, or treatment groups $(p>0.05)$. The vast majority of MSBs in the dlPFC formed two synaptic contacts ( 408 of $449,90.9 \%$ ), $8.46 \%$ (38 of 449) formed three contacts, and $0.67 \%$ ( 3 of 449) formed four contacts (seen only in 3 of 29 monkeys); MSBs with over four contacts were not observed.

\section{Relationship between working memory, MSBs, and mitochondrial morphology}

Last, we examined possible relationships between area 46 presynaptic measures and the integrity of working memory, as measured by DR average accuracy across 5-60 s delay intervals. Variability in working memory scores across young and aged premenopausal and postmenopausal subjects allowed for testing potential synaptic measures that are coupled to individual differ- ences in cognitive function known to depend on the dlPFC. Because of the limited number of subjects and the risk of overcorrection, bivariate correlation analyses were not corrected for multiple comparisons. However, the nine total significant correlations observed in this study greatly exceeded that predicted by chance $(1.05=21 \times 0.05)$.

First, Pearson correlation revealed a positive correlation between DR average accuracy and the frequency of MSBs $(n=29$; $r=0.403 ; p=0.03$; Fig. $4 A$ ), but not with the percentages of NSBs or SSBs $(p>0.05)$. This relationship between MSB frequency and working memory performance was pronounced when the analysis was confined to aged monkeys $(n=22 ; r=0.627 ; p=$ $0.002)$, but was not significant in the young group $(p>0.05)$. When axospinous and axodendritic MSBs were examined separately, working memory performance positively correlated with axospinous MSBs $(n=29 ; r=0.405 ; p=0.029)$, but not with axodendritic MSBs $(p>0.05)$. MSB frequency did not correlate with acquisition scores for the DR task (number of trials to criterion), or with performance on other tests of learning and memory (e.g., delayed nonmatching-to-sample test, $p>0.05$ for all; data not shown). Together, our data suggest that the observed 

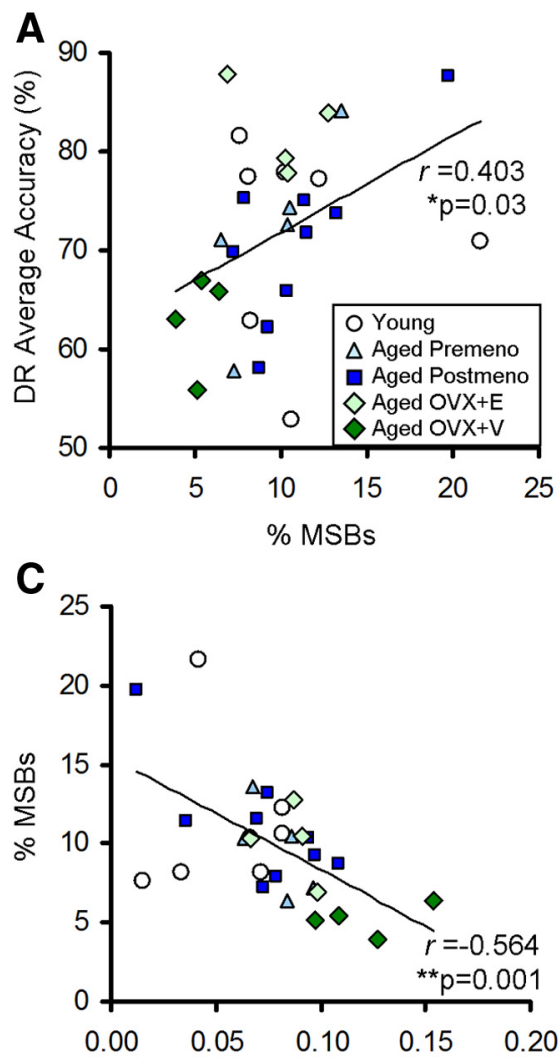

Avg \# of Donut Mitochondria / Bouton

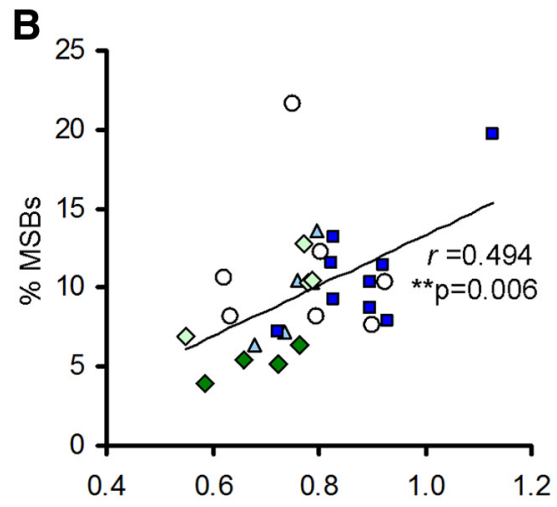

Avg \# of Straight Mitochondria / Bouton

D

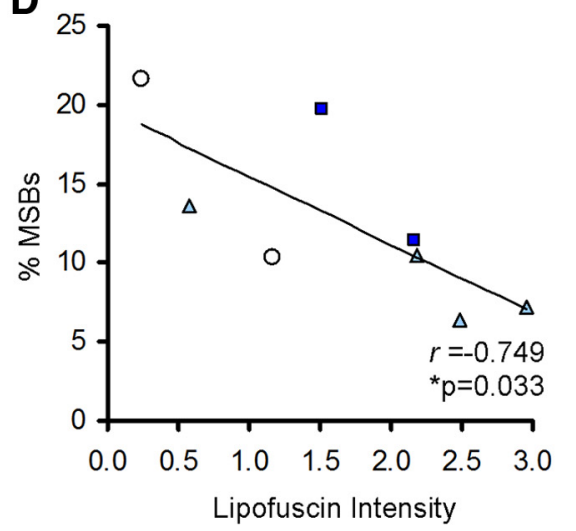

Figure 4. Relationships between working memory, MSBs, and mitochondrial morphology. $A, A$ significant positive correlation is seen between the proportion of MSBs and DR average accuracy. $B, C$, MSB frequency positively correlates with the average number of presynaptic straight mitochondria $(\boldsymbol{B})$ and inversely correlates with the average number of presynaptic donut-shaped mitochondria (C). D, MSB frequency inversely correlates with general oxidative damage in the dIPFC, as measured by lipofuscin autofluorescence intensity (arbitrary unit). For $\boldsymbol{A}-\boldsymbol{C}$, young, $n=7$; aged premenopausal, $n=5$; aged postmenopausal, $n=9$; aged $\mathrm{OVX}+\mathrm{E}, n=4$; aged $\mathrm{OVX}+\mathrm{V}, n=4$. For $\boldsymbol{D}$, young, $n=2$; aged premenopausal, $n=4$; aged postmenopausal, $n=2$.

correlations with MSBs are selective for dlPFC-associated working memory function.

As MSBs had larger volumes and contained more mitochondria, we examined the relationship between the frequency of MSBs and mitochondrial morphology. We reported previously that working memory performance positively correlates with the number of healthy straight mitochondria in area 46 boutons, whereas it inversely correlates with the number of malformed donut-shaped mitochondria (Hara et al., 2014). In addition, boutons containing donut-shaped mitochondria had smaller active zones and fewer docked synaptic vesicles compared to those housing straight mitochondria. Here, we observed that MSB frequency positively correlated with the average number of straight mitochondria per bouton $(n=29 ; r=0.494 ; p=0.006$; Fig. $4 B)$. This correlation was particularly strong when the analysis was confined to aged subjects $(n=22 ; r=0.750 ; p=0.00006)$ and was not significant in the young group $(p>0.05)$. This relationship was not driven by bouton size alone, as the average bouton volume failed to correlate with the number of total or straight mitochondria $(p>0.05)$, and the correlation between MSB frequency and straight mitochondria persisted after accounting for bouton volume (straight mitochondria/bouton volume; $n=29$; $r=0.489 ; p=0.024)$. In contrast, a significant inverse correlation was observed between MSB frequency and the average number of presynaptic donut-shaped mitochondria $(n=29 ; r=-0.564$; $p=0.001$; Fig. 4C), a morphological correlate of oxidative stress
(Liu and Hajnóczky, 2011). This correlation too, was robust when aged subjects were considered alone $(n=22 ; r=$ $-0.752 ; p=0.00005)$ and was not driven by bouton volume $(p>0.05)$.

Lipofuscin is an autofluorescent accumulation of nondegradable material largely composed of oxidatively damaged proteins and membrane lipids that, in turn, generates increased levels of reactive oxygen species (Terman and Brunk, 2006). Although not specific to presynaptic boutons, general oxidative damage in area 46 layer III was assessed using lipofuscin fluorescence intensity in 8 of the ovary-intact monkeys. Consistent with the strong relationship between MSBs and mitochondrial health, MSB frequency inversely correlated with the amount of lipofuscin $(n=8 ; r=-0.749 ; p=0.033$; Fig. 4D).

Together, our data suggest that maintenance of multisynaptic connections is energy demanding and is coupled to presynaptic mitochondrial health and is likely impeded by oxidative stress.

\section{Discussion}

MSB abundance was not altered by normal aging or natural menopause, but was significantly correlated with better working memory performance. In addition, MSB frequency decreased with surgical menopause (OVX) in aged monkeys but was restored with cyclic estradiol treatment (Fig. 5). Notably, the frequency of MSBs positively correlated with the number of healthy straight mitochondria and inversely correlated with the incidence of malformed donut-shaped mitochondria in dlPFC boutons. Together, our results suggest that MSBs are coupled to cognitive function and mitochondrial health and are sensitive to estrogen.

\section{Methodological considerations}

One limitation to our experimental design is that the ultrastructural data could only be obtained at a single time point, at postmortem, and therefore we were unable to establish a cause and effect relationship between our morphological data and the behavioral measures. However, the data we present here offer valuable information. Because both cognitive and morphological data were obtained from the same monkeys, we were able to test whether individual differences in working memory are coupled to select neurobiological parameters. In addition, we examined the effects of estrogen administration in aged ovariectomized animals to directly test whether a treatment known to improve working memory also influences presynaptic organization.

With regards to synaptic morphological analyses, serial section electron microscopy used in this study has major advantages over confocal, two-photon, and even single-section electron microscopy. The method used in this study is the only approach allowing high enough resolution in $3 \mathrm{D}$ for accurate and detailed 
assessment of synaptic organizations and mitochondrial morphology.

\section{Synaptic characteristics of monkey dlPFC axonal boutons: comparisons to hippocampal boutons}

We discovered that in the monkey dlPFC, only $10 \%$ of boutons was multisynaptic, and SSBs comprised the vast majority (over $80 \%$ ) regardless of age, menses status, or treatment. This percentage was markedly smaller compared to the monkey hippocampal dentate gyrus (DG), where $\sim 40 \%$ of boutons was multisynaptic (Hara et al., 2011). The maximum number of synaptic contacts per MSB was also different between these two brain regions. In the dlPFC, the majority of MSBs made only two contacts (90.9\%), and only 3 out of 29 monkeys exhibited an MSB forming four synaptic contacts, which was the highest observed in this brain region. In contrast, the maximum number of contacts observed in the monkey DG was six, and every single monkey had at least a few MSBs contacting four or more postsynaptic partners (Hara et al., 2011).

These findings may reflect the significantly lower spine density in the dIPFC compared to the DG (Dumitriu et al., 2010; Hara et al., $2012 \mathrm{~b}$ ) and suggest that synaptic connections in the dlPFC may be less complex, but more specific, with the majority of axonal boutons faithfully associated with a single postsynaptic partner. The distinct patterns of synaptic organization appear consistent with their differing functional roles. The dlPFC is critical in preserving spatiotemporal fidelity of ongoing experience (Funahashi et al., 1989; Wang et al., 2011). In contrast, the DG is involved in pattern separation, where small changes in the perforant path input from the entorhinal cortex can result in large changes in activation patterns of DG neurons (Neunuebel and Knierim, 2014; Newman and Hasselmo, 2014).

\section{Shifts in area 46 presynaptic connectivity with menopause: implications for natural versus surgical menopause}

One of our key findings was that MSBs in the dlPFC, and in particular axospinous MSBs, were sensitive to surgical menopause. Ovariectomized aged monkeys had less than half of these MSBs, whereas estradiol replacement in these monkeys restored MSB levels to those comparable to young and aged surgically intact premenopausal monkeys. The sensitivity of MSBs to estrogen is consistent with studies in the rat hippocampal CA1 (Woolley et al., 1996). In the rat CA1, the vast majority of MSBs form synapses with spines on multiple postsynaptic neurons rather than on spines within the same neurons (Yankova et al., 2001). If this pattern of synaptic connectivity is also present in MSBs of the monkey dlPFC, then surgical menopause may significantly reduce the level of complexity in the prefrontal neuronal circuit.

Interestingly, this drop in MSBs was not seen in monkeys that underwent natural menopause. Instead, NSBs were increased with natural menopause by over twofold. NSBs are capable of forming and releasing neurotransmitters (Henrikson and Vaughn, 1974; Prokop et al., 1996; Krueger et al., 2003) and are likely to be in transition into, or retraction from, functional connections (Gogolla et al., 2007). Thus, this increase in NSBs may suggest a menopause-related decrease in synaptic formation, stabilization, turnover, or a combination of all.

Collectively, our results are compatible with the literature suggesting that the abrupt loss of estrogen induced by surgical menopause results in morphological and molecular consequences distinct from those seen with natural menopause (Higaki et al., 2012; Hara et al., 2015). For example, we previously observed a significant increase in pathological donut-shaped mitochondria in dlPFC boutons of aged surgically menopausal monkeys that was not evident in naturally menopausal monkeys (Hara et al., 2014). These divergent trajectories between natural versus surgical menopause are also apparent in the clinical literature assessing cognitive functions. Women who undergo surgical menopause (oophorectomy) have an increased risk in developing cognitive impairment and dementia later in life (Sherwin, 1988; Rocca et al., 2007), whereas women undergoing the natural menopausal transition experience temporary and modest cognitive deficits (Henderson et al., 2003; Kok et al., 2006; Greendale et al., 2009; Epperson et al., 2013).

\section{Relationships between area 46 presynaptic connectivity, mitochondria, and working memory}

Although the incidence of MSBs in the dlPFC was not significantly different as a function of age, it positively correlated with the status of working memory, particularly among aged subjects. A previous rat study has shown that MSBs are increased in the hippocampal CA1 after associative learning (Geinisman et al., 2001). Our correlation with MSBs was specific to working memory function reliant on the integrity of dlPFC and not associated with hippocampus-dependent recognition memory. Thus, our finding is the first to document a potential role of MSBs in the $\mathrm{PFC}$ and its related cognitive functions.

We also revealed that the frequency of MSBs positively correlated with the average number of healthy straight mitochondria in dIPFC boutons and inversely correlated with the number of donut-shaped mitochondria, which are associated with oxidative stress (Liu and Hajnóczky, 2011). In addition, MSBs were signif- 
icantly larger than other bouton subtypes and contained predominantly healthy straight mitochondria, while curved and donut-shaped mitochondria were rarely present. These findings are consistent with our previous work showing that dlPFC boutons containing donut-shaped mitochondria formed abnormally small synaptic contacts, as indicated by small active zone areas and few docked synaptic vesicles (Hara et al., 2014). Our data suggest that sustaining multisynaptic connections may be energy demanding and may rely on abundant healthy mitochondria and low oxidative stress within the dlPFC. As observed in our ovariectomized monkeys, maintenance of MSBs may be especially challenging during menopause as perimenopausal women experience hypometabolism in cortical regions according to PET imaging studies (Rasgon et al., 2005; Rasgon et al., 2014; Brinton et al., 2015).

\section{Implications}

While our data demonstrate correlations and not causations, one potential hypothesis emerging from this work is that abundant healthy presynaptic mitochondria and low oxidative stress may support the formation and/or maintenance of MSBs that may, in turn, preserve working memory function during aging and menopause. Consistent with the wide variability of working memory performance exhibited by aged monkeys in our study and elsewhere (Bachevalier et al., 1991; Voytko and Tinkler, 2004), cognitive decline with aging or menopause is prevalent but not inevitable. Synaptic and metabolic dynamics within the dlPFC may be among the factors responsible for the cognitive reserve (Stern, 2012) or resilience (Terracciano et al., 2013) that confers protection against decline in many aged individuals. Thus, early interventions targeted at maintaining these parameters may offer effective protection against age- and menopauserelated cognitive dysfunction.

\section{References}

Arnsten AF, Wang MJ, Paspalas CD (2012) Neuromodulation of thought: flexibilities and vulnerabilities in prefrontal cortical network synapses. Neuron 76:223-239. CrossRef Medline

Bachevalier J, Landis LS, Walker LC, Brickson M, Mishkin M, Price DL, Cork LC (1991) Aged monkeys exhibit behavioral deficits indicative of widespread cerebral dysfunction. Neurobiol Aging 12:99-111. CrossRef Medline

Bartus RT, Fleming D, Johnson HR (1978) Aging in the rhesus monkey: debilitating effects on short-term memory. J Gerontol 33:858-871. CrossRef Medline

Brinton RD, Yao J, Yin F, Mack WJ, Cadenas E (2015) Perimenopause as a neurological transition state. Nat Rev Endocrinol 11:393-405. CrossRef Medline

Dumitriu D, Hao J, Hara Y, Kaufmann J, Janssen WG, Lou W, Rapp PR, Morrison JH (2010) Selective changes in thin spine density and morphology in monkey prefrontal cortex correlate with aging-related cognitive impairment. J Neurosci 30:7507-7515. CrossRef Medline

Epperson CN, Sammel MD, Freeman EW (2013) Menopause effects on verbal memory: findings from a longitudinal community cohort. J Clin Endocrinol Metab 98:3829-3838. CrossRef Medline

Funahashi S, Bruce CJ, Goldman-Rakic PS (1989) Mnemonic coding of visual space in the monkey's dorsolateral prefrontal cortex. J Neurophysiol 61:331-349. Medline

Geinisman Y, Berry RW, Disterhoft JF, Power JM, Van der Zee EA (2001) Associative learning elicits the formation of multiple-synapse boutons. J Neurosci 21:5568-5573. Medline

Gilardi KV, Shideler SE, Valverde CR, Roberts JA, Lasley BL (1997) Characterization of the onset of menopause in the rhesus macaque. Biol Reprod 57:335-340. CrossRef Medline

Gill S, Sharpless JL, Rado K, Hall JE (2002) Evidence that GnRH decreases with gonadal steroid feedback but increases with age in postmenopausal women. J Clin Endocrinol Metab 87:2290-2296. CrossRef Medline
Gogolla N, Galimberti I, Caroni P (2007) Structural plasticity of axon terminals in the adult. Curr Opin Neurobiol 17:516-524. CrossRef Medline

Goldman-Rakic PS (1995) Cellular basis of working memory. Neuron 14: 477-485. CrossRef Medline

Greendale GA, Huang MH, Wight RG, Seeman T, Luetters C, Avis NE, Johnston J, Karlamangla AS (2009) Effects of the menopause transition and hormone use on cognitive performance in midlife women. Neurology 72:1850-1857. CrossRef Medline

Hao J, Rapp PR, Janssen WG, Lou W, Lasley BL, Hof PR, Morrison JH (2007) Interactive effects of age and estrogen on cognition and pyramidal neurons in monkey prefrontal cortex. Proc Natl Acad Sci U S A 104:1146511470. CrossRef Medline

Hara Y, Park CS, Janssen WG, Punsoni M, Rapp PR, Morrison JH (2011) Synaptic characteristics of dentate gyrus axonal boutons and their relationships with aging, menopause, and memory in female rhesus monkeys. J Neurosci 31:7737-7744. CrossRef Medline

Hara Y, Rapp PR, Morrison JH (2012a) Neuronal and morphological bases of cognitive decline in aged rhesus monkeys. Age (Dordr) 34:1051-1073. CrossRef

Hara Y, Park CS, Janssen WG, Roberts MT, Morrison JH, Rapp PR (2012b) Synaptic correlates of memory and menopause in the hippocampal dentate gyrus in rhesus monkeys. Neurobiol Aging 33:421.e17-28. Medline

Hara Y, Punsoni M, Yuk F, Park CS, Janssen WG, Rapp PR, Morrison JH (2012c) Synaptic distributions of GluA2 and PKMzeta in the monkey dentate gyrus and their relationships with aging and memory. J Neurosci 32:7336-7344. CrossRef Medline

Hara Y, Yuk F, Puri R, Janssen WG, Rapp PR, Morrison JH (2014) Presynaptic mitochondrial morphology in monkey prefrontal cortex correlates with working memory and is improved with estrogen treatment. Proc Natl Acad Sci U S A 111:486-491. CrossRef Medline

Hara Y, Waters EM, McEwen BS, Morrison JH (2015) Estrogen effects on cognitive and synaptic health over the lifecourse. Physiol Rev 95:785-807. CrossRef Medline

Harris KM (1995) How multiple-synapse boutons could preserve input specificity during an interneuronal spread of LTP. Trends Neurosci 18 : 365-369. CrossRef Medline

Henderson VW, Guthrie JR, Dudley EC, Burger HG, Dennerstein L (2003) Estrogen exposures and memory at midlife: a population-based study of women. Neurology 60:1369-1371. CrossRef Medline

Henrikson CK, Vaughn JE (1974) Fine structural relationships between neurites and radial glial processes in developing mouse spinal cord. J Neurocytol 3:659-675. CrossRef Medline

Higaki S, Takumi K, Itoh M, Watanabe G, Taya K, Shimizu K, Hayashi M, Oishi T (2012) Response of ERbeta and aromatase expression in the monkey hippocampal formation to ovariectomy and menopause. Neurosci Res 72:148-154. CrossRef Medline

Kok HS, Kuh D, Cooper R, van der Schouw YT, Grobbee DE, Wadsworth ME, Richards M (2006) Cognitive function across the life course and the menopausal transition in a British birth cohort. Menopause 13:19-27. CrossRef Medline

Krueger SR, Kolar A, Fitzsimonds RM (2003) The presynaptic release apparatus is functional in the absence of dendritic contact and highly mobile within isolated axons. Neuron 40:945-957. CrossRef Medline

Liu X, Hajnóczky G (2011) Altered fusion dynamics underlie unique morphological changes in mitochondria during hypoxia-reoxygenation stress. Cell Death Differ 18:1561-1572. CrossRef Medline

Loerch PM, Lu T, Dakin KA, Vann JM, Isaacs A, Geula C, Wang J, Pan Y, Gabuzda DH, LiC, Prolla TA, Yankner BA (2008) Evolution of the aging brain transcriptome and synaptic regulation. PLoS One 3:e3329. CrossRef Medline

Matt DW, Kauma SW, Pincus SM, Veldhuis JD, Evans WS (1998) Characteristics of luteinizing hormone secretion in younger versus older premenopausal women. Am J Obstet Gynecol 178:504-510. CrossRef Medline

McCarthy G, Blamire AM, Puce A, Nobre AC, Bloch G, Hyder F, GoldmanRakic P, Shulman RG (1994) Functional magnetic resonance imaging of human prefrontal cortex activation during a spatial working memory task. Proc Natl Acad Sci U S A 91:8690-8694. CrossRef Medline

Neunuebel JP, Knierim JJ (2014) CA3 retrieves coherent representations from degraded input: direct evidence for CA3 pattern completion and dentate gyrus pattern separation. Neuron 81:416-427. CrossRef Medline 
Newman EL, Hasselmo ME (2014) CA3 sees the big picture while dentate gyrus splits hairs. Neuron 81:226-228. CrossRef Medline

Nichols SM, Bavister BD, Brenner CA, Didier PJ, Harrison RM, Kubisch HM (2005) Ovarian senescence in the rhesus monkey (Macaca mulatta). Hum Reprod 20:79-83. Medline

Oscar-Berman M, Hutner N, Bonner RT (1992) Visual and auditory spatial and nonspatial delayed-response performance by Korsakoff and nonKorsakoff alcoholic and aging individuals. Behav Neurosci 106:613-622. CrossRef Medline

Peters A, Sethares C, Luebke JI (2008) Synapses are lost during aging in the primate prefrontal cortex. Neuroscience 152:970-981. CrossRef Medline

Petrides M, Pandya DN (1999) Dorsolateral prefrontal cortex: comparative cytoarchitectonic analysis in the human and the macaque brain and corticocortical connection patterns. Eur J Neurosci 11:1011-1036. CrossRef Medline

Prokop A, Landgraf M, Rushton E, Broadie K, Bate M (1996) Presynaptic development at the Drosophila neuromuscular junction: assembly and localization of presynaptic active zones. Neuron 17:617-626. CrossRef Medline

Rapp PR, Amaral DG (1989) Evidence for task-dependent memory dysfunction in the aged monkey. J Neurosci 9:3568-3576. Medline

Rapp PR, Morrison JH, Roberts JA (2003) Cyclic estrogen replacement improves cognitive function in aged ovariectomized rhesus monkeys. J Neurosci 23:5708-5714. Medline

Rasgon NL, Silverman D, Siddarth P, Miller K, Ercoli LM, Elman S, Lavretsky H, Huang SC, Phelps ME, Small GW (2005) Estrogen use and brain metabolic change in postmenopausal women. Neurobiol Aging 26:229235. CrossRef Medline

Rasgon NL, Geist CL, Kenna HA, Wroolie TE, Williams KE, Silverman DH (2014) Prospective randomized trial to assess effects of continuing hormone therapy on cerebral function in postmenopausal women at risk for dementia. PLoS One 9:e89095. CrossRef Medline

Roberts JA, Gilardi KV, Lasley B, Rapp PR (1997) Reproductive senescence predicts cognitive decline in aged female monkeys. Neuroreport 8:20472051. CrossRef Medline

Rocca WA, Bower JH, Maraganore DM, Ahlskog JE, Grossardt BR, de Andrade M, Melton LJ 3rd (2007) Increased risk of cognitive impairment or dementia in women who underwent oophorectomy before menopause. Neurology 69:1074-1083. CrossRef Medline
Sherwin BB (1988) Estrogen and/or androgen replacement therapy and cognitive functioning in surgically menopausal women. Psychoneuroendocrinology 13:345-357. CrossRef Medline

Soghomonian JJ, Sethares C, Peters A (2010) Effects of age on axon terminals forming axosomatic and axodendritic inhibitory synapses in prefrontal cortex. Neuroscience 168:74-81. CrossRef Medline

Stern Y (2012) Cognitive reserve in ageing and Alzheimer's disease. Lancet Neurol 11:1006-1012. CrossRef Medline

Terman A, Brunk UT (2006) Oxidative stress, accumulation of biological 'garbage', and aging. Antioxid Redox Signal 8:197-204. CrossRef Medline

Terracciano A, Iacono D, O’Brien RJ, Troncoso JC, An Y, Sutin AR, Ferrucci L, Zonderman AB, Resnick SM (2013) Personality and resilience to Alzheimer's disease neuropathology: a prospective autopsy study. Neurobiol Aging 34:1045-1050. CrossRef Medline

Tigges J, Gordon TP, McClure HM, Hall EC, Peters A (1988) Survival rate and life span of rhesus monkeys at the Yerkes regional primate research center. Am J Primatol 15:263-273. CrossRef

Toni N, Buchs PA, Nikonenko I, Bron CR, Muller D (1999) LTP promotes formation of multiple spine synapses between a single axon terminal and a dendrite. Nature 402:421-425. CrossRef Medline

Voytko ML, Tinkler GP (2004) Cognitive function and its neural mechanisms in nonhuman primate models of aging, Alzheimer disease, and menopause. Front Biosci 9:1899-1914. CrossRef Medline

Walker ML, Herndon JG (2008) Menopause in nonhuman primates? Biol Reprod 79:398-406. CrossRef Medline

Wang M, Gamo NJ, Yang Y, Jin LE, Wang XJ, Laubach M, Mazer JA, Lee D, Arnsten AF (2011) Neuronal basis of age-related working memory decline. Nature 476:210-213. CrossRef Medline

Woller MJ, Everson-Binotto G, Nichols E, Acheson A, Keen KL, Bowers CY, Terasawa E (2002) Aging-related changes in release of growth hormone and luteinizing hormone in female rhesus monkeys. J Clin Endocrinol Metab 87:5160-5167. CrossRef Medline

Woolley CS, Wenzel HJ, Schwartzkroin PA (1996) Estradiol increases the frequency of multiple synapse boutons in the hippocampal CA1 region of the adult female rat. J Comp Neurol 373:108-117. Medline

Yankova M, Hart SA, Woolley CS (2001) Estrogen increases synaptic connectivity between single presynaptic inputs and multiple postsynaptic CA1 pyramidal cells: a serial electron-microscopic study. Proc Natl Acad Sci U S A 98:3525-3530. CrossRef Medline 